\title{
Le dépistage du cancer colorectal
}

État des lieux et perspectives 


\section{Springer}

Paris

Berlin

Heidelberg

New York

Hong Kong

Londres

Milan

Tokyo 
Étienne Dorval

\section{Le dépistage du cancer colorectal} État des lieux et perspectives

Springer 


\section{Étienne Dorval}

CHRU de Tours

Hôpital Trousseau

37044 Tours Cedex 1

ISBN-10 : 2-287-32802-5 Springer Paris Berlin Heidelberg New York ISBN-13 : 978-2-287-32802-2 Springer Paris Berlin Heidelberg New York

\section{(C) Springer-Verlag France, Paris 2006 \\ Imprimé en France}

\section{Springer-Verlag France est membre du groupe Springer Science + Business Media}

Cet ouvrage est soumis au copyright. Tous droits réservés, notamment la reproduction et la représentation, la traduction, la réimpression, l'exposé, la reproduction des illustrations et des tableaux, la transmission par voie d'enregistrement sonore ou visuel, la reproduction par microfilm ou tout autre moyen ainsi que la conservation des banques de données. La loi française sur le copyright du 9 septembre 1965 dans la version en vigueur n'autorise une reproduction intégrale ou partielle que dans certains cas, et en principe moyennant le paiement des droits. Toute représentation, reproduction, contrefaçon ou conservation dans une banque de données par quelque procédé que ce soit est sanctionnée par la loi pénale sur le copyright.

L'utilisation dans cet ouvrage de désignations, dénominations commerciales, marques de fabrique, etc. même sans spécification ne signifie pas que ces termes soient libres de la législation sur les marques de fabrique et la protection des marques et qu'ils puissent être utilisés par chacun.

La maison d'édition décline toute responsabilité quant à l'exactitude des indications de dosage et des modes d'emplois. Dans chaque cas il incombe à l'usager de vérifier les informations données par comparaison à la littérature existante.

SPIN : 11683513

Maquette de couverture : Jean-François Montmarché 


\section{Liste des auteurs}

Arber Nadir

Abolhassani Mohammad

Bejoul Bakthiar

Benamouzig Robert

Bretagne Jean-François

Caroli-Bosc François-Xavier
Unité de prévention des cancers

Service de gastro-entérologie

Tel Aviv Sourasky Medical Center, Tel Aviv University

6 Weizmann Street, Tel Aviv 64239, Israël

Service d'hépato-gastro-entérologie

CHU Henri-Mondor

51, avenue du Maréchal de Lattre de Tassigny

94010 Créteil, France

Unité de prévention des cancers

Service de gastro-entérologie

Tel Aviv Sourasky Medical Center, Tel Aviv University

6 Weizmann Street, Tel Aviv 64239, Israël

Service de gastro-entérologie

Hôpital Avicenne

125 , rue de Stalingrad

93009 Bobigny Cedex, France

Service des maladies de l'appareil digestif

CHU Pontchaillou

2, rue Henri-le-Guilloux

35033 Rennes, France

Service de gastro-entérologie

CHU Nice

Route Saint-Antoine Ginestière

BP 3079

06202 Nice Cedex, France 
Dorval Étienne

Faivre Jean

Heresbach Denis

Lejeune Catherine

Lepage Côme

Mitry Emmanuel

Sobhani Iradj
Service de Gastro-entérologie

CHU Trousseau

37044 Tours Cedex 1, France

Registre bourguignon des cancers digestifs

(INSERM EMI 0106)

Faculté de médecine

7, boulevard Jeanne d'Arc

BP 87900

21079 Dijon Cedex, France

Service des maladies de l'appareil digestif

CHU Pontchaillou

2, rue Henri-le-Guilloux

35033 Rennes, France

Registre bourguignon des cancers digestifs

(INSERM EMI 0106)

Faculté de médecine

7, boulevard Jeanne d'Arc

BP 87900

21079 Dijon Cedex, France

Registre bourguignon des cancers digestifs (INSERM EMI 0106)

Faculté de médecine

7, boulevard Jeanne d'Arc

BP 87900

21079 Dijon Cedex, France

Service d'hépato-gastro-entérologie

et oncologie digestive

CHU Ambroise-Paré

9, avenue Charles-de-Gaulle

92100 Boulogne, France

Service d'hépato-gastro-entérologie

CHU Henri-Mondor

51, avenue du Maréchal de Lattre de Tassigny

94010 Créteil, France 


\section{Sommaire}

Rationnel du dépistage

Emmanuel Mitry

Mise en place du dépistage organisé du cancer colorectal en France

Côme Lepage et Jean Faivre

Tests fécaux de dépistage du cancer colorectal en dehors de l'Hemoccult II ${ }^{\circledR}$ et des tests moléculaires

François-Xavier Caroli-Bosc et Étienne Dorval

Dépistage des tumeurs colorectales par les tests moléculaires $\quad$... 43 Iradj Sobhani et Mohammad Abolhassani

Dépistage du cancer colorectal par endoscopie classique ou coloscopie virtuelle

Denis Heresbach et Jean-François Bretagne

Dépistage du cancer colorectal : quelles techniques endoscopiques pour demain?

Robert Benamouzig, Bakthiar Bejoul et Nadir Arber

Approche médico-économique du dépistage

du cancer colorectal

Catherine Lejeune 